Bull. Korean Math. Soc. 50 (2013), No. 2, pp. 499-506

http://dx.doi.org/10.4134/BKMS.2013.50.2.499

\title{
UNIQUENESS OF THE SOLUTION OF HALF INVERSE PROBLEM FOR THE IMPULSIVE STURM LIOUVILLE OPERATOR
}

\author{
A. Sinan Ozkan, Baki Keskin, and Yasar Cakmak
}

\begin{abstract}
The half-inverse spectral problem for an impulsive SturmLiouville operator consists in reconstruction of this operator from its spectrum and half of the potential. In this study, the spectrum of the impulsive Sturm-Liouville problem is given and by using the Hochstadt and Lieberman's method we show that if $q(x)$ is prescribed on $\left(0, \frac{\pi}{2}\right)$, then only one spectrum is sufficient to determine $q(x)$ on the interval $(0, \pi)$ for this problem
\end{abstract}

\section{Introduction}

Assume that $Q(x)$ is a real-valued function in $L_{2}(0, \pi), \alpha$ and $\beta$ are real numbers such that $0<\alpha<1$ and $\beta>0$. Define $\rho(x)=\left\{\begin{array}{c}1, \quad x<\frac{\pi}{2} \\ \alpha^{2}, \quad x>\frac{\pi}{2}\end{array}\right.$ and denote by $L=L(Q(x), \rho(x), \beta)$ a Sturm-Liouville problem in $L_{2}(0, \pi)$ that is given by the differential equation

$$
\ell y:=-y^{\prime \prime}(x)+Q(x) y(x)=\lambda \rho(x) y(x), \quad x \in\left(0, \frac{\pi}{2}\right) \cup\left(\frac{\pi}{2}, \pi\right)
$$

with the boundary conditions

$$
\begin{aligned}
& U(y):=y^{\prime}(0)=0 \\
& V(y):=y^{\prime}(\pi)=0
\end{aligned}
$$

and the jump conditions

$$
\left\{\begin{aligned}
y\left(\frac{\pi}{2}+0\right) & =\beta y\left(\frac{\pi}{2}-0\right) \\
y^{\prime}\left(\frac{\pi}{2}+0\right) & =\beta^{-1} y^{\prime}\left(\frac{\pi}{2}-0\right),
\end{aligned}\right.
$$

where $\lambda$ is the spectral parameter.

Received October 25, 2011; Revised February 22, 2012.

2010 Mathematics Subject Classification. 34A55, 34B24, 34L05, 45C05.

Key words and phrases. Sturm-Liouville operator, determination of the potential, discontinuous condition, half inverse problem. 
The equation (1) appears in some physical problems. For example, an equation for vibration in a solid $0<x<\pi$, composed of a layer $0<x<\frac{\pi}{2}$ of material in contact with a layer $\frac{\pi}{2}<x<\pi$ of another material, is given by,

$$
p(x) \frac{\partial^{2} u(x, s)}{\partial s^{2}}=\frac{\partial^{2} u(x, s)}{\partial x^{2}}+q(x) u(x, s), \quad x \in\left(0, \frac{\pi}{2}\right) \cup\left(\frac{\pi}{2}, \pi\right), s>0,
$$

where, $p(x)$ is a piecewise continuous function in $\left(0, \frac{\pi}{2}\right) \cup\left(\frac{\pi}{2}, \pi\right)$ which has the form $p(x)=\left\{\begin{array}{cc}1, & 0<x<\frac{\pi}{2} \\ \alpha^{-2}, & \frac{\pi}{2}<x<\pi\end{array}\right.$ (see [1] and [11]). By using the classical Fourier method, an equation like (1) can be obtained.

Spectral problems for Sturm-Liouville operators were extensively well studied in [2], [3], [8] and [10].

Boundary value problems with discontinuities inside the interval often appear in mathematics, mechanics, physics, geophysics and other branches of natural properties. The jump conditions like (4) appear in some important physical problem. [5] is well known work about discontinuous inverse eigenvalue problems.

Half-inverse problem for a Sturm-Liouville operator consists in reconstruction of this operator from its spectrum and half of the potential. The first result on the half-inverse problem is due to Hochstadt and Lieberman [6]. Later, Hald generalized a theorem in [5]. In [4] and [9] some new uniqueness results in inverse spectral analysis with partial information on the potential for scalar and matrix Sturm-Liouville equations were given, respectively. In 2001, the existence of solution to the half-inverse problem was studied by Sakhnovich [12]. In [7], half-inverse problems were solved for Sturm-Liouville operators with singular potentials. In this work, authors gave the necessary and sufficient condition for solvability of the half-inverse spectral problem for the class of Sturm-Liouville operators with singular potentials from the space $W_{2}^{-1}(0,1)$. In [13], a half-inverse problem was solved for the Sturm-Liouville equation with eigenparameter dependent boundary conditions. In [14], a similar problem was solved for Dirac operator by using Hochstadt and Lieberman's method.

In the present paper, we consider a Sturm-Liouville operator with jump conditions inside the interval. The aim of this work is to solve half-inverse problem by using the Hochstadt and Lieberman's method for this kind of operator. We will prove that the coefficients of the boundary value problem can be uniquely determined by the potential in $\left(0, \frac{\pi}{2}\right)$ and a single spectrum.

\section{Preliminaries}

Let $\varphi(x, \lambda)$ be the solution of (1), satisfies the initial conditions $\varphi(0, \lambda)=1$, $\varphi^{\prime}(0, \lambda)=0$ and jump conditions (4).

It is clear that the equation (1) can be written as follows

$$
-\frac{1}{\sqrt{\rho(x)}}\left(\frac{1}{\sqrt{\rho(x)}} y^{\prime}\right)^{\prime}+q(x) y=\lambda y, \quad x \in\left(0, \frac{\pi}{2}\right) \cup\left(\frac{\pi}{2}, \pi\right) .
$$


Here $q(x)=\frac{Q(x)}{\rho(x)}$. Therefore, the function $\varphi(x, \lambda)$ satisfies the following integral equation for $x<\frac{\pi}{2}$

$$
\varphi(x, k)=\cos k \gamma(x)+\int_{0}^{x} \frac{\sin k(\gamma(x)-\gamma(t))}{k} \sqrt{\rho(t)} q(t) \varphi(t, k) d t,
$$

where $\gamma(x)=\int_{0}^{x} \sqrt{\rho(t)} d t, k=\sqrt{\lambda}$. If the solution $\varphi(x, \lambda)$ is continued to the interval $\left(\frac{\pi}{2}, \pi\right)$ as

$$
\begin{aligned}
\varphi(x, k)= & A(k) \cos k \gamma(x)+B(k) \sin k \gamma(x) \\
& +\int_{0}^{x} \frac{\sin k(\gamma(x)-\gamma(t))}{k} \sqrt{\rho(t)} q(t) \varphi(t, k) d t,
\end{aligned}
$$

then from the jump condition (4) we obtain

$$
\begin{aligned}
\varphi(x, k)= & \beta^{+} \cos k \gamma(x)+\beta^{-} \cos k\left(2 \gamma\left(\frac{\pi}{2}\right)-\gamma(x)\right) \\
& +\frac{1}{k} \int_{0}^{\frac{\pi}{2}} \beta^{+} \sin k(\gamma(x)-\gamma(t)) \sqrt{\rho(t)} q(t) \varphi(t, k) d t \\
& +\frac{1}{k} \int_{0}^{\frac{\pi}{2}} \beta^{-} \sin k\left(2 \gamma\left(\frac{\pi}{2}\right)-\gamma(x)-\gamma(t)\right) \sqrt{\rho(t)} q(t) \varphi(t, k) d t \\
& +\frac{1}{k} \int_{\frac{\pi}{2}}^{x} \sin k(\gamma(x)-\gamma(t)) \sqrt{\rho(t)} q(t) \varphi(t, k) d t, \quad x>\frac{\pi}{2} .
\end{aligned}
$$

Here $\beta^{ \pm}=\frac{1}{2}\left(\beta \pm \frac{1}{\alpha \beta}\right)$.

It is easily verified from above integral equations that the following asymptotic relations are valid as $|k| \rightarrow \infty$ :

$$
\begin{aligned}
\varphi(x, k)= & \cos k \gamma(x)+O\left(\frac{1}{k} \exp |\tau| \gamma(x)\right) \quad \text { for } x<\frac{\pi}{2}, \\
\varphi(x, k)= & \beta^{+} \cos k \gamma(x)+\beta^{-} \cos k\left(2 \gamma\left(\frac{\pi}{2}\right)-\gamma(x)\right) \\
& +O\left(\frac{1}{k} \exp |\tau| \gamma(x)\right) \quad \text { for } x>\frac{\pi}{2} .
\end{aligned}
$$

Now, let the function $\psi(x, \lambda)$ be the solution of $(1)$, which satisfies the initial conditions $\psi(\pi, \lambda)=1, \quad \psi^{\prime}(\pi, \lambda)=0$ and jump conditions (4). The function

$$
\Delta(\lambda)=W[\varphi, \psi]=-\varphi^{\prime}(\pi, \lambda)=\psi^{\prime}(0, \lambda)
$$

is called the characteristic function of the problem $L$. Let $\left\{\lambda_{n}\right\}$ be the zeros of $\Delta(\lambda)$. It is clear that the numbers $\lambda_{n}$ coincide with the eigenvalues of the problem $L$. From (7) and (8) we have

$$
\begin{aligned}
\Delta(\lambda)= & \alpha \lambda^{\frac{1}{2}}\left[-\beta^{+} \sin \sqrt{\lambda} \frac{\pi}{2}(1+\alpha)+\beta^{-} \sin \sqrt{\lambda} \frac{\pi}{2}(1-\alpha)\right] \\
& +O(\exp |\tau| \gamma(\pi)) .
\end{aligned}
$$


Note that for $\lambda \in G_{\epsilon}:=\left\{\lambda:\left|\lambda-\lambda_{n}\right|>\varepsilon\right\}$,

$$
|\Delta(\lambda)| \geq C_{1}|\lambda|^{\frac{1}{2}} \exp |\tau| \gamma(\pi),
$$

where $\varepsilon$ is sufficiently small number. The following theorem can be proved by using same methods in [2] and [15].

Theorem 1. The eigenvalues $\left\{\lambda_{n}\right\}_{n \geq 0}$ of the problem $L$ are real, simple and have the following asymptotics

$$
\sqrt{\lambda_{n}}=\frac{2 n}{1+\alpha}\left[1+O\left(\frac{1}{n}\right)\right]
$$

for $n \rightarrow \infty$.

The following representation holds (see [2])

(12) $\psi(x, \lambda)=\cos \alpha \sqrt{\lambda}(\pi-x)+\int_{x}^{\pi} K(x, t) \cos \alpha \sqrt{\lambda}(\pi-t) d t \quad$ for $x>\frac{\pi}{2}$,

where $K(x, t)$ is a continuous function which does not depend on $\lambda$.

\section{Main results}

Now we state the main result of this work. It is assumed in what follows that if a certain symbol $s$ denotes an object related to $L$, then the corresponding symbol $\widetilde{s}$ with tilde denote the analogous object related to $\widetilde{L}$. Let us denote by $\varphi\left(x, \lambda_{n}\right)$, the eigenfunction which corresponds to $\lambda_{n}$.

Lemma 1. If $\lambda_{n}=\widetilde{\lambda}_{n}$ for all $n \in \mathbb{N}$, then $\rho(x)=\widetilde{\rho}(x)$ and $\beta=\widetilde{\beta}$.

Proof. From (11), a direct calculation yields $\alpha=\widetilde{\alpha}$, i.e., $\rho(x)=\widetilde{\rho}(x)$.

Now, we shall prove that $\beta=\widetilde{\beta}$. Since $\lambda_{n}=\widetilde{\lambda}_{n} ; \Delta(\lambda)$ and $\widetilde{\Delta}(\lambda)$ are entire functions in $\lambda$ of order $\frac{1}{2}$, by Hadamard's factorization theorem,

$$
\Delta(\lambda)=C \widetilde{\Delta}(\lambda)
$$

is valid for all $\lambda \in \mathbb{C}$.

Denote $\Delta_{0}(\lambda):=\alpha \lambda^{\frac{1}{2}}\left[-\beta^{+} \sin \sqrt{\lambda} \frac{\pi}{2}(1+\alpha)+\beta^{-} \sin \sqrt{\lambda} \frac{\pi}{2}(1-\alpha)\right]$, and we can write

$$
\Delta_{0}(\lambda)-C \widetilde{\Delta}_{0}(\lambda)=C\left[\widetilde{\Delta}(\lambda)-\widetilde{\Delta}_{0}(\lambda)\right]-\left[\Delta(\lambda)-\Delta_{0}(\lambda)\right] .
$$

It follows from (9) and (14) that

$$
\begin{aligned}
& C\left[\widetilde{\Delta}(\lambda)-\widetilde{\Delta}_{0}(\lambda)\right]-\left[\Delta(\lambda)-\Delta_{0}(\lambda)\right] \\
= & \alpha \lambda^{\frac{1}{2}}\left\{\left[-\beta^{+} \sin \sqrt{\lambda} \frac{\pi}{2}(1+\alpha)+\beta^{-} \sin \sqrt{\lambda} \frac{\pi}{2}(1-\alpha)\right]\right. \\
& \left.-C\left[-\widetilde{\beta}^{+} \sin \sqrt{\lambda} \frac{\pi}{2}(1+\alpha)+\widetilde{\beta}^{-} \sin \sqrt{\lambda} \frac{\pi}{2}(1-\alpha)\right]\right\} .
\end{aligned}
$$


Multiplying both sides of (15) by $\sin k \frac{\pi}{2}(1+\alpha)$ and integrating with respect to $k$ in $(0, T)$ with any positive real number $T$, we get

$$
\begin{aligned}
& \int_{0}^{T} \frac{k^{-1}}{\alpha}\left\{C\left[\widetilde{\Delta}(k)-\widetilde{\Delta}_{0}(k)\right]-\left[\Delta(k)-\Delta_{0}(k)\right]\right\} \sin k \frac{\pi}{2}(1+\alpha) d k \\
(16)= & \int_{0}^{T}\left\{\left[-\beta^{+} \sin k \frac{\pi}{2}(1+\alpha)+\beta^{-} \sin k \frac{\pi}{2}(1-\alpha)\right] \sin k \frac{\pi}{2}(1+\alpha) d k\right. \\
& -C \int_{0}^{T}\left[-\widetilde{\beta}^{+} \sin k \frac{\pi}{2}(1+\alpha)+\widetilde{\beta}^{-} \sin k \frac{\pi}{2}(1-\alpha)\right] \sin k \frac{\pi}{2}(1+\alpha) d k .
\end{aligned}
$$

Since $\widetilde{\Delta}(k)-\widetilde{\Delta}_{0}(k)=O(1)$ and $\left[\Delta(k)-\Delta_{0}(k)\right]=O(1)$ for $k$ in $(0, T)$, direct calculation in (16) yields,

$$
C \widetilde{\beta}^{+}-\beta^{+}=O\left(T^{-1}\right) .
$$

By letting $T \rightarrow \infty$, we conclude that

$$
\beta^{+}=C \widetilde{\beta}^{+} .
$$

Similarly, if we multiply both sides of $(15)$ by $\sin k \frac{\pi}{2}(1-\alpha)$ and integrate again with respect to $k$ on $(0, T)$, we get

$$
\beta^{-}=C \widetilde{\beta}^{-} .
$$

(18) and (19) imply that $C^{2}=1$, but since $\beta^{+}$and $\widetilde{\beta}^{+}$are positive we conclude that $C=1$. Hence, $\beta=\widetilde{\beta}$. This completes the proof.

Theorem 2. If $\lambda_{n}=\widetilde{\lambda}_{n}$ for all $n \in \mathbb{N}$ and $Q(x)=\widetilde{Q}(x)$ on $\left[0, \frac{\pi}{2}\right]$, then $Q(x)=\widetilde{Q}(x)$ almost everywhere on $[0, \pi]$.

Proof. It follows from (12) that

$$
\psi(x, \lambda) \widetilde{\psi}(x, \lambda)=\frac{1}{2}\left[1+\cos 2 \alpha \sqrt{\lambda}(\pi-x)+\int_{x}^{\pi} V(x, t) \cos 2 \alpha \sqrt{\lambda}(\pi-t) d t\right],
$$

where $V(x, t)$ is a continuous function which does not depend on $\lambda$.

Let us write the equation

$$
-\psi^{\prime \prime}(x, \lambda)+Q(x) \psi(x, \lambda)=\lambda \rho(x) \psi(x, \lambda)
$$

and

$$
-\widetilde{\psi}^{\prime \prime}(x, \lambda)+\widetilde{Q}(x) \widetilde{\psi}(x, \lambda)=\lambda \widetilde{\rho}(x) \psi(x, \lambda) .
$$

Multiplying (21) and (22) by $\widetilde{\psi}(x, \lambda)$ and $-\psi(x, \lambda)$, respectively, and adding together we obtain

$$
\begin{aligned}
& \widetilde{\psi}^{\prime \prime}(x, \lambda) \psi(x, \lambda)-\psi^{\prime \prime}(x, \lambda) \widetilde{\psi}(x, \lambda)+(Q(x)-\widetilde{Q}(x)) \psi(x, \lambda) \widetilde{\psi}(x, \lambda) \\
= & \lambda(\rho(x)-\widetilde{\rho}(x)) \psi(x, \lambda) \widetilde{\psi}(x, \lambda) .
\end{aligned}
$$


Taking Lemma 1 into account we get

$$
\widetilde{\psi}^{\prime \prime}(x, \lambda) \psi(x, \lambda)-\psi^{\prime \prime}(x, \lambda) \widetilde{\psi}(x, \lambda)+(Q(x)-\widetilde{Q}(x)) \psi(x, \lambda) \widetilde{\psi}(x, \lambda)=0 .
$$

After integrating this equality on $[0, \pi]$, from the hypothesis $Q(x)=\widetilde{Q}(x)$ on $\left[0, \frac{\pi}{2}\right]$, we find

$$
\begin{aligned}
& \int_{\pi / 2}^{\pi}[\widetilde{Q}(x)-Q(x)] \psi(x, \lambda) \widetilde{\psi}(x, \lambda) d x \\
= & {\left[\psi(x, \lambda) \widetilde{\psi}^{\prime}(x, \lambda)-\psi^{\prime}(x, \lambda) \widetilde{\psi}(x, \lambda)\right]\left(\left.\right|_{0} ^{\pi / 2}+\left.\right|_{\pi / 2} ^{\pi}\right) } \\
= & -\psi(0, \lambda) \widetilde{\psi}^{\prime}(0, \lambda)-\psi^{\prime}(0, \lambda) \widetilde{\psi}(0, \lambda) .
\end{aligned}
$$

Define

$$
H(\lambda):=\int_{\pi / 2}^{\pi}[\widetilde{Q}(x)-Q(x)] \psi(x, \lambda) \widetilde{\psi}(x, \lambda) d x .
$$

It is clear from (23) that $H\left(\lambda_{n}\right)=0$ for each eigenvalue $\lambda_{n}$. Therefore $\chi(\lambda):=$ $\frac{H(\lambda)}{\Delta(\lambda)}$ is entire in $\lambda$. We obtain from (12) and (24) that $|H(\lambda)| \leq C \exp (\tau \alpha \pi)$. Thus, from (10) we get

$$
|\chi(\lambda)| \leq C|\lambda|^{-\frac{1}{2}} \exp \left[\tau \frac{\pi}{2}(\alpha-1)\right] .
$$

Since $0<\alpha<1, \chi(\lambda)=O\left(\lambda^{-\frac{1}{2}}\right)$ for sufficiently large $|\lambda|$. Hence by Liouville's theorem $\chi(\lambda)=0$ for all $\lambda$. This means $H(\lambda)=0$.

Define $\Omega(x)=\widetilde{Q}(x)-Q(x)$. We get on the whole $\lambda$-plane,

$$
\begin{aligned}
& \int_{\frac{\pi}{2}}^{\pi} \Omega(x)(1+\cos 2 \sqrt{\lambda}(\pi-x)) d x \\
& +\int_{\frac{\pi}{2}}^{\pi} \Omega(x)\left[\int_{x}^{\pi} V(x, t) \cos 2 \sqrt{\lambda}(\pi-t) d t\right] d x=0 .
\end{aligned}
$$

This equation can be written as

$$
\int_{\frac{\pi}{2}}^{\pi} \Omega(x) d x+\int_{\frac{\pi}{2}}^{\pi} \cos 2 \sqrt{\lambda}(\pi-t)\left[\Omega(t)+\int_{\frac{\pi}{2}}^{t} \Omega(x) V(x, t) d x\right] d t=0 .
$$

Letting $\lambda \rightarrow \infty$ for real $\lambda$ in (25), we see from Riemann-Lebesque lemma that

$$
\int_{\frac{\pi}{2}}^{\pi} \Omega(x) d x=0
$$

and so

$$
\int_{\frac{\pi}{2}}^{\pi} \cos 2 \sqrt{\lambda}(\pi-t)\left[\Omega(t)+\int_{\frac{\pi}{2}}^{t} \Omega(x) V(x, t) d x\right] d t=0
$$


Therefore, it follows from the completeness of the function $\cos 2 \sqrt{\lambda}(\pi-x)$ in $L_{2}\left(\frac{\pi}{2}, \pi\right)$ that

$$
\Omega(t)+\int_{\frac{\pi}{2}}^{t} \Omega(x) V(x, t) d x=0, t \in\left(\frac{\pi}{2}, \pi\right) .
$$

Since the equation (26) is a homogenous Volterra integral equation, it has only trivial solution. Hence, we have obtained $\Omega(x)=\widetilde{Q}(x)-Q(x)=0$ on $\left(\frac{\pi}{2}, \pi\right)$, i.e., $Q(x)=\widetilde{Q}(x)$, almost everywhere on $\left(\frac{\pi}{2}, \pi\right)$. This completes the proof.

Corollary 1. The problem $L(Q(x), \rho(x), \beta)$ is uniquely determined by the sequence $\left\{\lambda_{n}\right\}_{n \geq 0}$ under the assumption $Q(x)=\widetilde{Q}(x)$ on $\left[0, \frac{\pi}{2}\right]$.

\section{References}

[1] P. E. Bulavin and V. M. Kascheev, Solution of the nonhomogeneous heat conduction equation for multilayered bodies, Int. Chem. Engng. 5 (1965), 112-115.

[2] G. Freiling and V. A. Yurko, Inverse Sturm-Liouville Problems and Their Applications, Huntington, NY: Nova Science, 2001.

[3] I. M. Gelfand and B. M. Levitan, On the determination of a differential equation from its spectral function, Amer. Math. Transl. 1 (1951), no. 2, 239-253.

[4] F. Gesztesy and B. Simon, Inverse spectral analysis with partial information on the potential II: The case of discrete spectrum, Trans. Amer. Math. Soc. 352 (2000), no. 6, 2765-2787.

[5] O. H. Hald, Discontinuous inverse eigenvalue problem, Comm. Pure Appl. Math. 37 (1984), no. 5, 539-577.

[6] H. Hochstadt and B. Lieberman, An inverse Sturm-Liouville problem with mixed given data, SIAM J. Appl. Math. 34 (1978), no. 4, 676-680.

[7] O. R. Hryniv and Y. V. Mykytyuk, Half-inverse spectral problems for Sturm-Liouville operators with singular potentials, Inverse Problems 20 (2004), no. 5, 1423-1444.

[8] B. M. Levitan, and I. S. Sargsyan, Sturm-Liouville and Dirac Operators Kluwer Academic Publishers, Dodrecht/Boston/London, 1991.

[9] M. M. Malamud, Questions of uniqueness in inverse problems for systems of differential equations on a finite interval, Tr. Mosk. Mat. Obs. 60 (1999), 199-258; translation in Trans. Moscow Math. Soc. (1999), 173-224.

[10] V. A. Marchenko, Sturm-Liouville Operators and Their Applications, Birkhauser, 1986.

[11] M. N. Ozisik, Boundary Value Problems of Heat Conduction, Dower, New York, 1989.

[12] L. Sakhnovich, Half inverse problems on the finite interval, Inverse Problems 17 (2001), no. 3, 527-532.

[13] C.-F. Yang and Z.-Y. Huang, A half-inverse problem with eigenparameter dependent boundary conditions, Numer. Funct. Anal. Optim. 31 (2010), no. 6, 754-762.

[14] C.-F. Yang, Hochstadt-Lieberman theorem for Dirac operator with eigenparameter dependent boundary conditions, Nonlinear Anal. 74 (2011), no. 7, 2475-2484.

[15] V. F. Ždanovič, V. F., Formulae for the zeros of Dirichlet polynomials and quasipolynomials, Dokl. Acad. Nauk SSSR 135 (1960), no. 8, 1046-1049.

\section{A. SINAN OZKAN}

Department of Mathematics Faculty of Art \& Sci.

Cumhuriyet University

58140 Sivas, TuRkEY

E-mail address: sozkan@cumhuriyet.edu.tr 


\section{BAKI KESKIN}

Department of Mathematics Faculty of Art \& Sci.

Cumhuriyet University

58140 Sivas, TURKEY

E-mail address: bkeskin@cumhuriyet.edu.tr

YASAR CAKMAK

Department of Mathematics Faculty of Art \& Sci.

Cumhuriyet University

58140 Sivas, TuRkEY

E-mail address: ycakmak@cumhuriyet.edu.tr 